\title{
A quantitative study of Cooperative Awareness Messages in production VANETs
}

\author{
Jakob Breu ${ }^{1,2^{*}}$, Achim Brakemeier ${ }^{2}$ and Michael Menth ${ }^{1}$
}

\begin{abstract}
In Vehicular Ad-hoc Networks (VANETs), vehicles gather Cooperative Awareness Messages (CAMs) sent from other vehicles via wireless broadcast. Each received message has to be processed by an in-vehicle system. In series implementations, such an in-vehicle system needs to cope with limited resources whose capacity is not yet defined. Therefore, information about the received CAM rate is a crucial input for the development of series VANET products. CAMs from distant vehicles are less likely to be received than those of nearby vehicles. Designers of applications leveraging CAM information are interested in the frequency of received CAMs originating from vehicles depending on their distance. In this paper, we study future CAM rates depending on various parameters. We set up a road traffic simulation for selected highway scenarios. We estimate the rates of generated CAMs and introduce the notion of relative channel load. We present a new approximative channel model to determine a vehicle's message reception probability. That model is used to simulate the rates of received CAMs for each vehicle. Moreover, we investigate the origin of received CAMs and times between consecutive CAMs received from the same sender (inter-reception times) depending on distance. Most results depend on the penetration rate of VANET technology that will increase in the near future. We derive approximative formulae and use them to validate our simulation results. They are quite accurate, and so they may also serve for simple forecasts. The results from our analysis show that the rates of generated and received CAMs lead to several challenges for the design of an efficient and robust VANET implementation.
\end{abstract}

Keywords: VANET; Cooperative Awareness Messages; Traffic analysis

\section{Introduction}

Wireless communication among vehicles and between vehicles and roadside infrastructure has been identified [1] as a key technology to enhance road traffic safety, advance driving comfort, and increase ecological and economic efficiency. Typical terms for these efforts are Vehicular Ad-hoc Networks (VANETs), Car-to-Car communication (Car2Car, C2C), or Vehicle-to-Vehicle communication $(V 2 V)$.

Currently, VANET technology is on the leap to market introduction. A set of standards for a first implementation will be finished soon, and harmonization among continents is taking place. Multiple field tests of different extent are conducted and show the practicability of these standards [2]. In the context of this work, the main result

${ }^{*}$ Correspondence: jakob.breu@student.uni-tuebingen.de

1 Department of Computer Science, University of Tübingen, Tübingen 72074 Germany

${ }^{2}$ Mercedes-Benz Research and Development, Daimler AG, Sindelfingen 71063, Germany of the European standardization is the use of two types of messages: event-based messages and status information messages $[3,4]$. The latter is sent frequently by each participating vehicle to inform receivers about their environment and to support various applications. They are called Cooperative Awareness Messages (CAMs). A CAM is sent only when a set of rules is met so that the resulting CAM send frequency can be 1 to $10 \mathrm{~Hz}$. The CAMs are aggregated in a so-called local dynamic map (LDM). From this database, information about the traffic situation can be extracted.

In recently conducted experiments and field operational tests, the abundant processing power for the low rate of received CAMs was more than sufficient. However, in series vehicles, this will be challenging for several reasons. The hardware is embedded and provides less computing capabilities than the powerful hardware that is usually installed in experimental vehicles. Also, the field operational tests have a limited number of participating vehicles

\section{望 Springer}

(c) 2014 Breu et al: licensee Springer. This is an Open Access article distributed under the terms of the Creative Commons Attribution License (http://creativecommons.org/licenses/by/2.0), which permits unrestricted use, distribution, and reproduction in any medium, provided the original work is properly credited. 
and are focused on message processing at lower networking layers. Therefore, a bottleneck in the processing chain could not be detected so far. However, the CAM load in in-vehicle systems will increase with the penetration rate of VANET technology in series vehicles. Initial market introductions are expected for 2015 according to a memorandum of understanding among European car manufacturers [5]. Each VANET system that is sold has to be able to cope with rising penetration rates for its complete lifetime [6,7]. This is a challenge and the motivation for this study of CAMs in future VANETs.

Our methodology is as follows. We set up a road traffic simulation for two selected highway scenarios. First, we derive the rate of CAMs which are generated by each vehicle based on its movement. Second, we introduce a new statistical channel model which computes the probability for a successful message reception based on distance between sender and receiver, and a newly defined relative channel load. This allows the calculation of the rate of CAMs received by vehicles equipped with VANET technology. We use the statistical channel model to give approximation formulae to validate the results of the simulation. We use these formulae to conduct parameter studies of the relative channel load and received CAM rates. Finally, we use our simulation and analytic estimations to quantify the frequency of CAMs received from a certain vehicle depending on distance and characterize the local origin of all received CAMs.

The remainder of this paper is organized as follows. Table 1 provides an overview of the abbreviations and symbols used in this paper. In Section 2, we briefly review related work. Section 3 gives an introduction to the standardized message types and their triggers. The core Sections 4 and 8 are structured as depicted in Figure 1. Section 4 presents the simulation setup and scenarios used to evaluate the rate of generated CAMs. In Section 5, we introduce a new statistical channel model to derive the reception probability of a CAM. In Section 6, we investigate the channel load by extending our simulation and using an estimating formula, which we also use in Section 7 to determine the rates of received CAMs. Section 8 describes an analysis of the origins of received CAMs. In Section 9, we evaluate times between consecutive CAMs received from the same vehicle (interreception times) depending on its distance. Finally, in Section 10, we conclude and provide an outlook on further research.

\section{Related work}

Standards for VANET technologies are in the finalization process for a first market introduction. In parallel, national and international field operational tests are conducted. Their aim is to prove the interoperability and practical feasibility of the standards. Three
Table 1 List of abbreviations and symbols

\begin{tabular}{|c|c|}
\hline $\begin{array}{l}\text { Abbreviation/ } \\
\text { symbol }\end{array}$ & Meaning \\
\hline A5, A6 & Autobahn 5/6 \\
\hline$B_{C A M}$ & CAM size (bytes) \\
\hline C & Transmission bandwidth of $802.11 p$ \\
\hline C2C, Car2Car & Car-to-car communication \\
\hline CAM & Cooperative awareness message \\
\hline$d_{\mathrm{co}}$ & Crossover distance \\
\hline$d_{\text {range }}^{\text {comm }}$ & Communication range (radius) \\
\hline DENM & Decentralized environmental notification message \\
\hline $\begin{array}{l}\text { inter } \\
\text { vehicle }\end{array}$ & Average distance between vehicles on a lane \\
\hline$d_{\mathrm{HS}}$ & Hidden station range (radius) \\
\hline$d$ & Distance between sender and receiver \\
\hline$\gamma$ & Nakagami signal attenuation parameter \\
\hline$\Gamma_{\text {SNR }}$ & Signal-to-noise ratio \\
\hline$h_{\mathrm{snd}}, h_{\mathrm{rcv}}$ & Antenna height of sender and receiver \\
\hline Ivehicle & Average length of vehicles \\
\hline$\lambda$ & Signal wavelength \\
\hline LDM & Local dynamic map \\
\hline$\mu$ & Path loss coefficient \\
\hline$n_{\text {lanes }}$ & Number of overall parallel lanes of a road \\
\hline$n_{\text {revd }}^{\text {passing }}$ & $\begin{array}{l}\text { Number of received CAMs from a single vehicle while } \\
\text { passing }\end{array}$ \\
\hline$\varphi$ & Penetration rate of VANET technology \\
\hline$P_{\left[d_{1}, d_{2}\right]}$ & $\begin{array}{l}\text { Average message reception probability for distance interval } \\
{\left[d_{1}, d_{2}\right]}\end{array}$ \\
\hline $\begin{array}{l}\text { Puccess } \\
\text { combined }\end{array}$ & Overall CAM reception probability \\
\hline $\begin{array}{l}\text { Nuccess } \\
\text { Nakagami } \\
\end{array}$ & Message reception probability for Nakagami model \\
\hline $\begin{array}{l}\text { loss } \\
\text { hidden }\end{array}$ & Message loss probability due to hidden stations \\
\hline $\begin{array}{r}\text { revd } \\
\text { revd }\end{array}$ & Rate of received CAMs $\left(\frac{C A M s}{s}\right)$ \\
\hline$r_{\text {sent }}^{\mathrm{cr}}$ & Rate of sent CAMs within communication range of receiver \\
\hline$r_{\mathrm{rcvd}}^{\mathrm{cr}}$ & $\begin{array}{l}\text { Rate of received CAMs within communication range of } \\
\text { receiver }\end{array}$ \\
\hline$\rho$ & Relative channel load \\
\hline$\sigma_{\text {CAM }}$ & CAM generation density per meter and second \\
\hline$\sigma_{\text {vehicle }}$ & Vehicle density $\left(\frac{\text { vehicles }}{m}\right)$ \\
\hline SUMO & Simulator for Urban MObility \\
\hline$T_{\text {air }}$ & Air-time of message on channel \\
\hline$T_{C A}$ & Channel access time \\
\hline $\begin{array}{l}\text { Sent } \\
\end{array}$ & Inter-generation time of CAMs from a single sender \\
\hline$T_{d}^{\text {inter }}$ & Inter-reception time of CAMs from senders with distance $d$ \\
\hline$T_{\left[d_{1}, d_{2}\right]}^{\text {inter }}$ & $\begin{array}{l}\text { Inter-reception time of CAMs from senders with distance } d_{1} \\
\text { to } d_{2}\end{array}$ \\
\hline$T_{\text {Off }}$ & Back-off time for CAM triggering \\
\hline$t_{\text {pass }}$ & $\begin{array}{l}\text { Communication time between vehicles passing in opposite } \\
\text { direction }\end{array}$ \\
\hline $\mathrm{TraCl}$ & Traffic control interface \\
\hline VANET & Vehicular ad-hoc network \\
\hline$v$ & Average vehicle speed \\
\hline V2V & Vehicle-to-vehicle communication \\
\hline
\end{tabular}




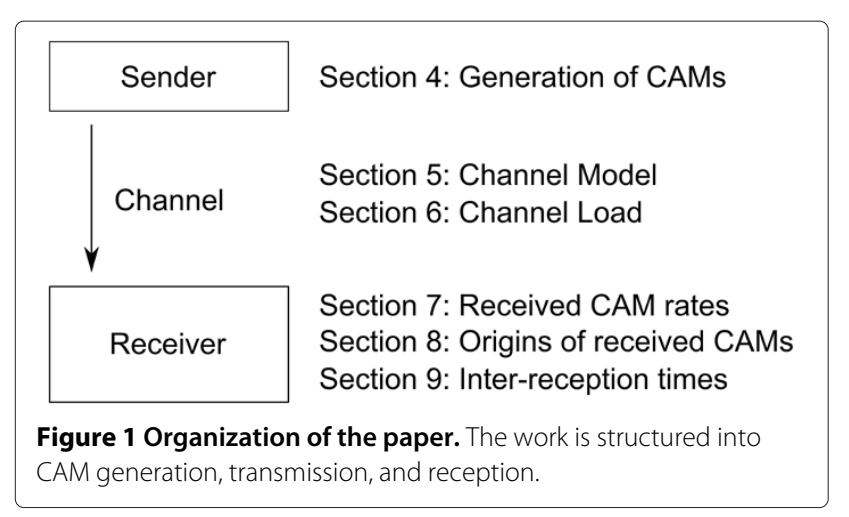

recently conducted field operational tests were realized in Germany ( $\operatorname{sim}^{\mathrm{TD}}$ ), France (score@F), and Europe (DRIVE C2X) [8-10]. As they focused on the communication between vehicles, these field operational tests utilized powerful hardware. For implementation in series vehicles, less powerful hardware will be used for size, cost, and robustness reasons.

In academic research on the topic of VANETs, complete tool chains for simulations are usually used. Examples for such tool chains are VEINS and VSimRTI [11,12]. Instead of applying one of these products, we chose to build our own tool chain. This simplifies the use of our new statistical channel model and the controllability of all simulation modules. Nevertheless, we take advantage of available tools like the road simulator SUMO [13].

Several papers report investigations of radio transmission quality in real experiments $[14,15]$. In general, lineof-sight is one key for a high message reception probability. Therefore, we focus on highway scenarios on parallel lanes where line-of-sight between communicating vehicles can be assumed. In contrast, highway interchanges are places with a very high number of vehicles and high message sending rates, but the radio transmission quality between the street levels is low.

Statistical channel models for reception of VANET messages have been published in $[16,17]$ based on studies in [18]. We adapt them by incorporating a simple representation for the hidden station effect into the channel model. This effect was identified to be the dominant one for multiple sender scenarios in a non-congested channel [19].

Characteristics of message reception in VANETs have been gathered in several publications, e.g., [20-23]. The results depend on the setup of the conducted experiment or simulation, the tools applied, usage of recent standards, and overall objectives. To our knowledge, there is no approach available that combines a statistical channel model and available European VANET standards and estimates channel load, rates of received CAMs, interreception times between CAMs from a vehicle depending on distance, and the spatial distribution of the origin of received CAMs. These performance metrics are required for the sizing and design of a CAM-processing architecture in series vehicles and for developers of applications using CAM information.

\section{VANET message types and message triggers}

The European Telecommunications Standards Institute (ETSI) defined two message types to support VANET applications: Decentralized Environmental Notification Messages (DENMs) and Cooperative Awareness Messages (CAMs). We briefly review their purpose and describe under which conditions they are triggered.

\subsection{Decentralized Environmental Notification Message}

DENMs are used by vehicles and roadside infrastructure to notify other traffic participants about specific events on or near the roads. Each DENM is the result of an event detection process conducted by the sending vehicle, and therefore, DENMs occur infrequently and rarely [3]. Because of their rare occurrence, DENMs do not create a large processing load at the receiver. For this reason, we can neglect them in our following investigations.

\subsection{Cooperative Awareness Messages}

To enable vehicles to be aware of their environment, all vehicles and some road infrastructure devices in a VANET broadcast CAMs [4]. CAMs contain information about the sender like vehicle type, position, heading, speed, and acceleration. Each participating vehicle has to send CAMs on a regular basis. The receiver of such messages interprets them and creates a so-called local dynamic map (LDM). The LDM is an environment database maintained in and by each vehicle and supports various applications.

The quality of the environment awareness correlates with the number of CAMs received from traffic participants. However, unnecessary status updates from many senders, e.g., for standing vehicles, could lead to channel congestion. Therefore, CAMs are only triggered when a set of rules is met [3]:

- The current heading of the vehicle differs at least $4^{\circ}$ from the heading in the last CAM, or

- The current position of the vehicle differs at least $4 \mathrm{~m}$ from the position in the last CAM, or

- The current speed of the vehicle differs at least $0.5 \frac{\mathrm{m}}{\mathrm{s}}$ from the speed in the last CAM, or

- The last CAM was sent $1 \mathrm{~s}$ earlier.

Each of these conditions has to be checked repeatedly after a back-off time $T_{\text {Off }}[3,24]$. Usually $T_{\text {Off }}$ is set to $100 \mathrm{~ms}$. To avoid channel congestion, $T_{\mathrm{Off}}$ is set to higher values if the relative channel load exceeds $25 \%$. This ultimately leads to lower CAM generation frequencies. 


\section{Simulative approximation of CAM generation}

In this section, we describe the setup for our road traffic simulation and introduce two realistic road traffic scenarios. The road traffic simulation generates movement traces for all simulated vehicles. We use these traces to derive the instances of CAM transmissions. They yield a characterization of CAM inter-generation times, and they are input to the channel model in the next section.

\subsection{Road traffic simulation}

Our tool chain utilizes the microscopic and continuous traffic simulator SUMO for vehicle trace generation [13]. We chose SUMO because of its convenience, features, and wide acceptance in the field of VANET simulation [25]. Moreover, SUMO is computationally efficient even for big simulation scenarios [26].

Since SUMO's ability to save vehicle traces into files is limited, we chose to use the traffic control interface (TraCI) to connect a Java program via TCP connection to SUMO. This approach allows us to get all vehicle information we need and save it to a file on disk for later use [27].

Figure 2 illustrates our simulation tool chain from the map extraction to the output of the vehicle trajectory dump: We extracted a road map from OpenStreetMap via jOSM, which we converted with the SUMO tool netconvert to a network file. This network file and a route file created with the tool traffic modeler is the input for SUMO [28]. Our own tool TraCI Dump saves the vehicle trajectories of the traffic simulation to a file. This file is then used by a CAM triggering program to generate logs which contain information about all generated CAMs.

\subsection{Derivation of CAM generation instances}

Based on the vehicle trajectory dump created with SUMO, we execute the CAM triggering algorithm as described in Section 3.2. For each simulation time step, a check is performed whether the conditions for the generation of a new CAM are met, as long as a vehicle has not left the simulated map section. Each CAM and its generation time is logged and accumulated for statistical analysis.

\subsection{Road traffic simulation scenarios}

We chose two typical German map sections: one from the Autobahn A5 south of Frankfurt having eight lanes in parallel (see Figure 3) and the other from the Autobahn A6 near Nuremberg having the typical four parallel lanes on German highways (see Figure 4). Both Autobahn sections have low crash barriers and no walls between the lanes. We assume the VANET antennas to be mounted on top of the vehicles and, therefore, can postulate line-of-sight for message transmission on the wireless channel.

The traffic simulator was parameterized in a way that vehicle departs, speeds, and routes ensure a high vehicle density and high vehicle speeds. The road sections for traffic simulation also contain highway interchanges and ramps. This ensures typical road traffic entropy and helps to avoid homogeneous and unnatural effects in vehicular movement. We limit the area for statistical analyses to highway areas between the interchanges. Statistics for receivers are gathered only within an area with a distance of at least $1 \mathrm{~km}$ to the intersections.

Our simulations cover about 8,000 and 6,000 vehicles on the A5 and A6 scenario within a time frame of $1 \mathrm{~h}$. The simulation proceeds in time steps of $100 \mathrm{~ms}$ so that time is measured as a multiple of $100 \mathrm{~ms}$ in the simulation results.

\subsection{Numerical results}

We first illustrate and analyze the dependence of distance between vehicles, CAM inter-generation times, and CAM rate density on vehicle speeds.

\subsubsection{Dependency of CAM generation on vehicle speeds}

As CAM generation rules take vehicle dynamics into account, the CAM generation rate per vehicle increases with the vehicle's speed. Furthermore, the average distance between vehicles increases with speed [29]. As a result, the CAM rate density, i.e., the CAM rate generated per road segment and time interval, also depends on the average vehicle speed. We illustrate these relationships in the following.

Figure 5 shows the average inter-vehicle distance $d_{\text {vehicle }}^{\text {inter }}$. For safety reasons, it increases with increasing speed [29]. The figure also shows how the time between consecutively

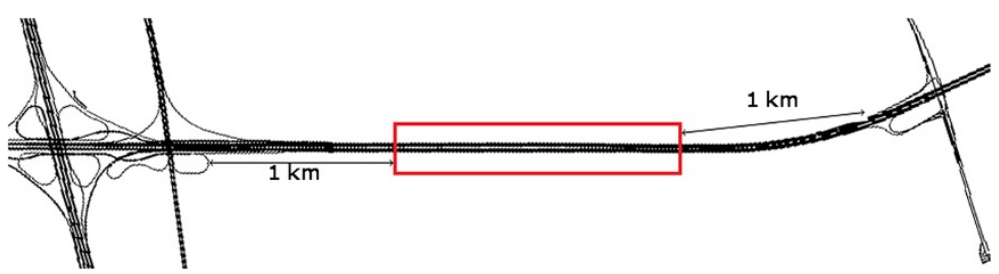

Figure 2 Overview of the simulation tool chain. 


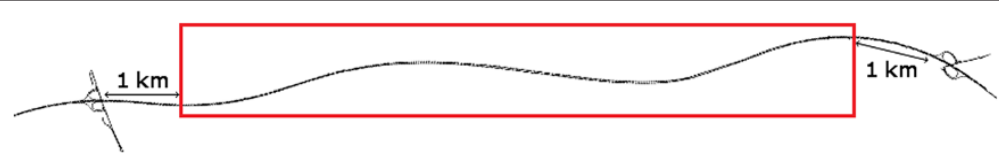

Figure 3 Simulated road section A5. Autobahn A5 south of Frankfurt, Germany. CAMs received within the boxed areas are used for the statistics.

generated CAMs by each vehicle $T_{\text {CAM }}^{\text {inter }}$ decreases with increasing speed $v$ of that vehicle. To determine the speed for which we can expect the highest CAM rates on the transmission channel, we define the CAM rate density as

$$
\begin{aligned}
\sigma_{\mathrm{CAM}} & =\frac{n_{\text {lanes }} \cdot \sigma_{\text {vehicle }}}{T_{\mathrm{CAM}}^{\text {inter }}} \text { with } \\
\sigma_{\text {vehicle }} & =\frac{1}{d_{\text {vehicle }}^{\text {inter }}+l_{\text {vehicle }}}
\end{aligned}
$$

The parameter $n_{\text {lanes }}$ is the overall number of lanes in both directions, and $l_{\text {vehicle }}$ indicates the average vehicle

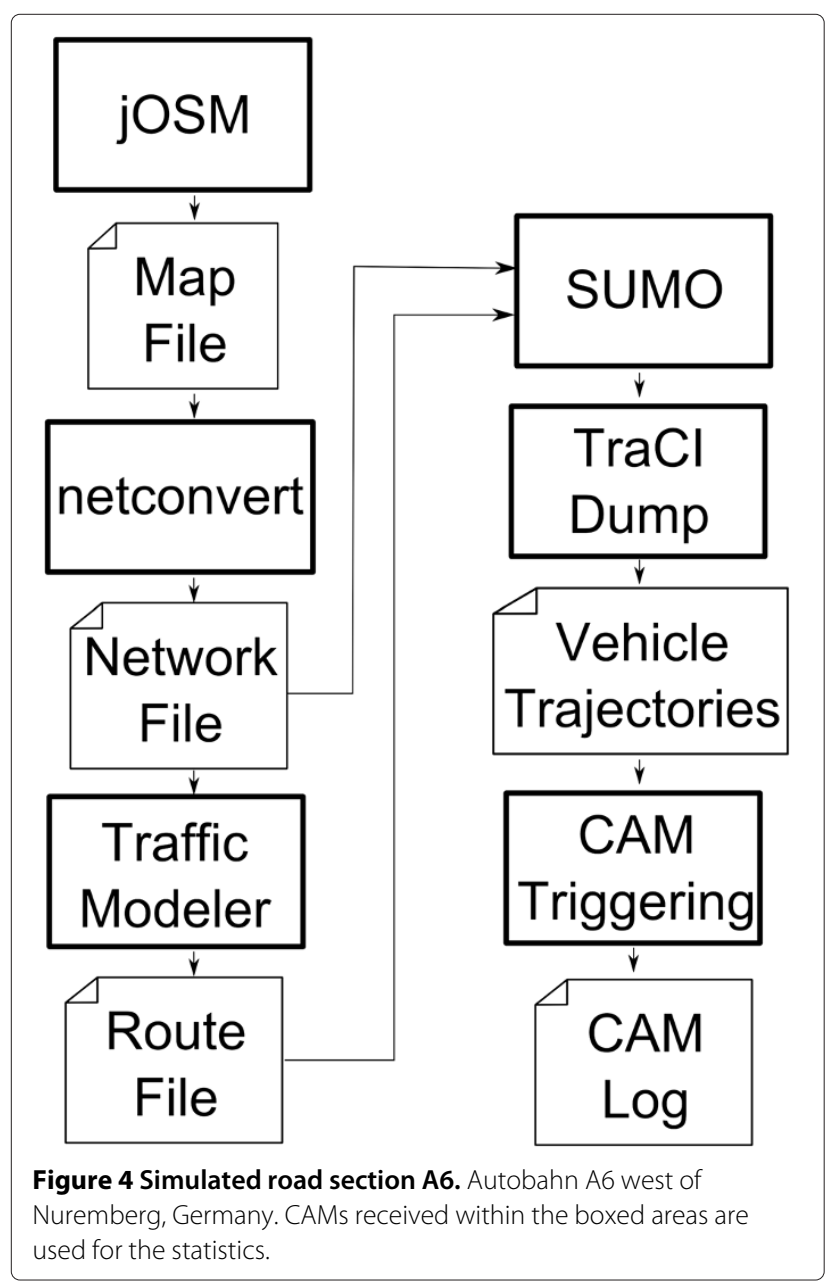

length so that $\sigma_{\text {vehicle }}$ is the vehicle density on a single lane in terms of number of vehicles per meter. Multiplying the CAM rate density $\sigma_{\text {CAM }}$ with a road distance yields the CAM rate generated on that road section. Figure 6 shows the CAM rate density of a single lane. It first increases and then decreases with increasing speed so that we observe a maximum CAM rate density at a speed of $72 \frac{\mathrm{km}}{\mathrm{h}}$. Therefore, we chose that speed to run our simulations in the following.

\subsubsection{CAM inter-generation times}

Figure 7 shows a histogram of CAM inter-generation times from vehicles in our simulation. The time between most consecutive CAMs is $200 \mathrm{~ms}$. This is a characteristic value for free or synchronized traffic on highways where the typical cruise speed is 72 to $144 \frac{\mathrm{km}}{\mathrm{h}}$. At that speed, vehicles change their position by more than $4 \mathrm{~m}$ after 200 ms which triggers the generation of a CAM, yielding that value a typical CAM inter-generation time.

\section{Channel approximation model}

In this section, we introduce a new statistical channel model. It will be used to compute the reception probability of a message depending on the distance between sender and receiver and the current relative channel load. We use the statistical channel model to simulate the reception of each generated CAM by other vehicles. We do not use discrete-event network simulators like ns-2 or OMNet++ because of their complexity and huge set of tunable parameters. The statistical channel model provides the most relevant characteristics of the radio channel for our purposes.

First, we define the notion of relative channel load. Then, we derive the new statistical channel model by explaining its main components: signal attenuation for a single sender-receiver pair and the hidden station phenomenon which is the dominating effect in the presence of multiple senders and low channel loads [19].

\subsection{Definition of relative channel load}

A vehicle's antenna obtains signals from all senders within its maximum achievable communication range with radius $d_{\text {range }}^{\text {comm }}$. The rate of generated CAMs from vehicles within this range is denoted by $r_{\text {sent }}^{\mathrm{cr}}$. The fraction of $r_{\text {sent }}^{\mathrm{cr}}$ and the transmission bandwidth $C$ define the 


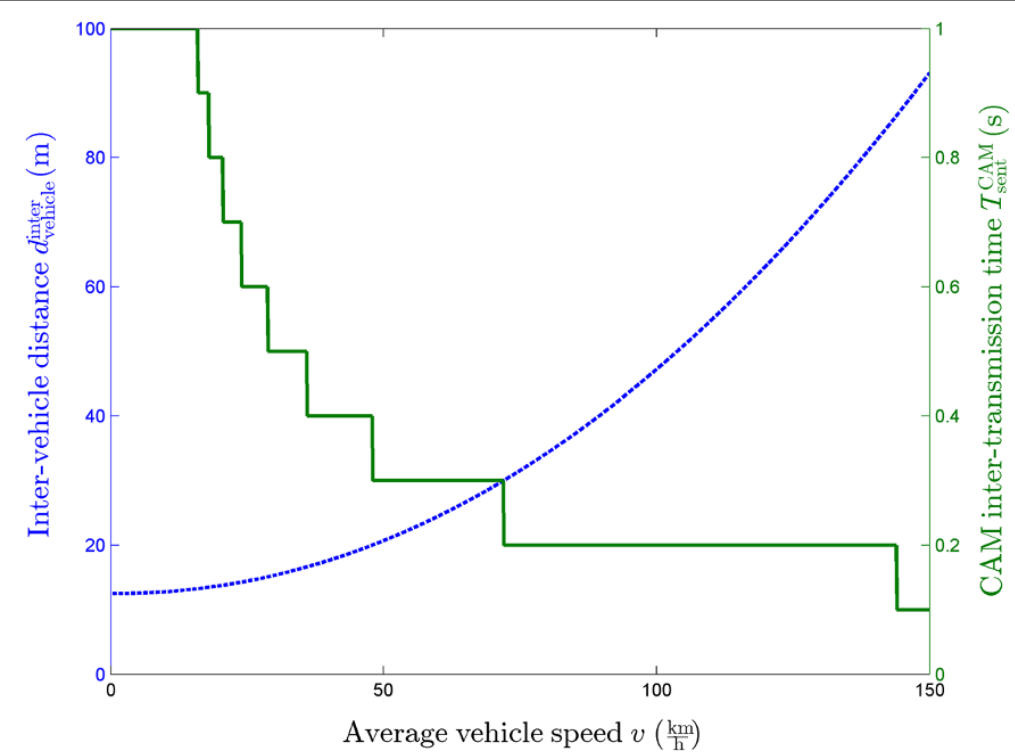

Figure 5 Impact of vehicle speed. The CAM inter-generation time and the inter-vehicle distance depend on the average vehicle speed.

channel load for message size $B_{\mathrm{CAM}}$ :

$$
\rho=\frac{r_{\text {sent }}^{\mathrm{cr}} \cdot B_{\mathrm{CAM}}}{C}
$$

We use the default transmission bandwidth of 802.11p, i.e., $C=6 \frac{\mathrm{Mbit}}{\mathrm{s}}$, and a message size of $B_{\mathrm{CAM}}=200$ bytes $[4,30]$.
5.2 Signal attenuation for a single sender and receiver The Nakagami $m$-distribution is a well-known model to accurately characterize signal attenuation in wireless channels [31]. This holds also for VANETs and was experimentally validated for typical setups [18]. Killat et al. used a tuned model of the Nakagami $m$-distribution to provide a function for the reception probability of a message depending on the distance $d$ between sender and receiver.

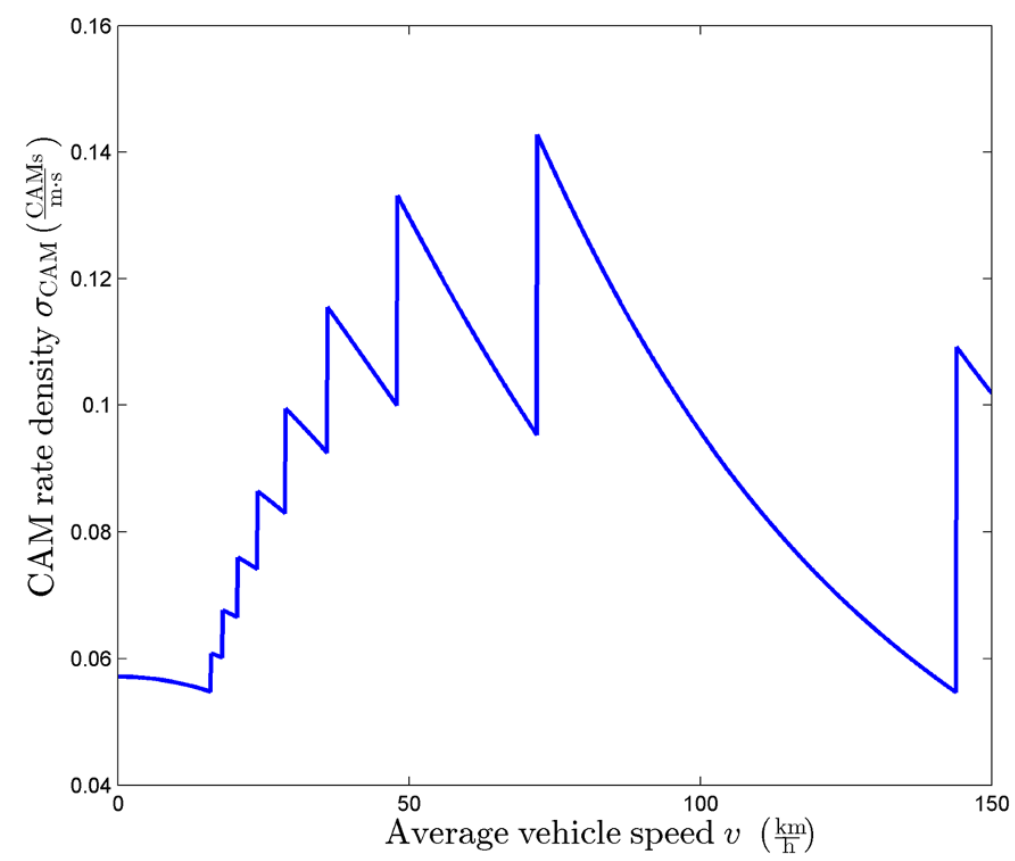

Figure 6 CAM rate density for a single lane. The CAM rate density $\sigma_{\text {CAM }}$ depends on the average vehicle speed $v$. 


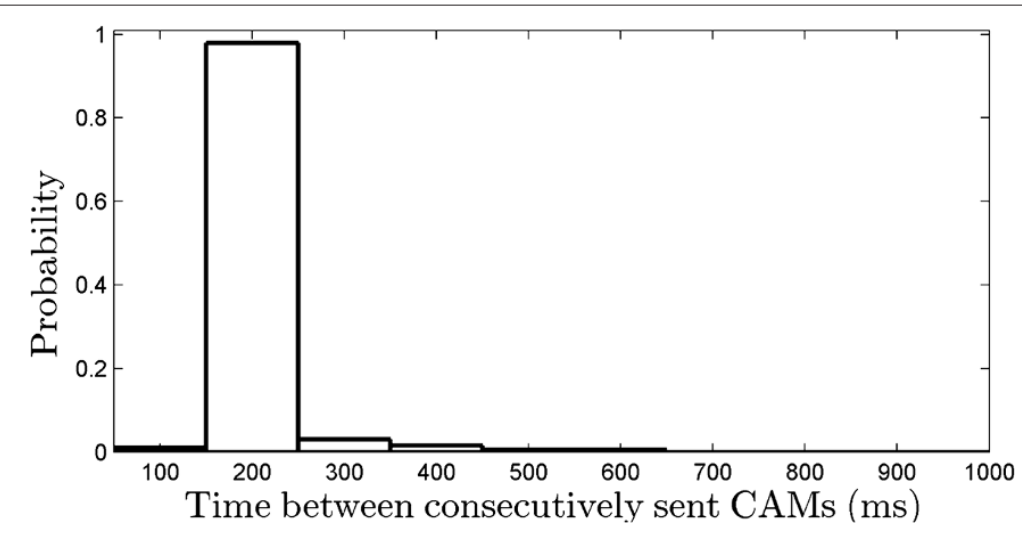

Figure 7 CAM inter-generation time. Histogram of time $T_{C A M}^{\text {inter }}$ between consecutive CAMs generated by vehicles in the simulation.

They validated their model using a discrete-event network simulation [16,17].

The model divides the communication range $d_{\text {range }}^{\text {comm }}$ in two parts. For small distances, the model considers Friis path loss, while for higher distances, it considers the tworay ground model. The transition distance is denoted as the crossover distance

$$
d_{\mathrm{co}}=4 \cdot \pi \cdot \frac{h_{\mathrm{snd}} \cdot h_{\mathrm{rcv}}}{\lambda},
$$

where $h_{\text {snd }}$ and $h_{\mathrm{rcv}}$ are the installation heights of the sender's and receiver's antennas and $\lambda$ is the wavelength of the signal $[32,33]$. For distances greater than $d_{\text {co }}$, the signal reflection on the ground as formulated by the two-ray ground model needs to be respected.

They derived the following formula for the transmission success rate:

$$
\begin{aligned}
& P_{\text {Nakagami }}^{\text {success }}(d)= \\
& \left\{\begin{array}{c}
e^{-3\left(\frac{d}{d_{\text {range }}^{\text {commm }}}\right)^{2}} \cdot\left(1+2\left(\frac{d}{d_{\text {range }}^{\text {conm }}}\right)^{2}+\frac{9}{2}\left(\frac{d}{d_{\text {range }}^{\text {comm }}}\right)^{4}\right), \\
\text { if } d \leq d_{c o} \\
e^{-3 \gamma\left(\frac{d^{2}}{d_{\text {ramge }}^{\text {conm }}}\right)^{2}} \cdot\left(\begin{array}{c}
\left.1+2 \gamma\left(\frac{d^{2}}{d_{\text {ramge }}^{\text {comm }}}\right)^{2}+\frac{9}{2} \gamma^{2}\left(\frac{d^{2}}{d_{\text {range }}}\right)^{4}\right) \\
\text { if } d>d_{c o} .
\end{array}\right.
\end{array}\right.
\end{aligned}
$$

The parameter $\gamma$ depends on the height of both sender and receiver antennas and the wavelength of the signal, and can be calculated as

$$
\gamma=\left(\frac{\lambda}{4 \cdot \pi \cdot h_{\mathrm{snd}} \cdot h_{\mathrm{rcv}}}\right)^{2}=\left(d_{c o}\right)^{-2} .
$$

\subsection{Hidden station phenomenon for multiple senders}

Because of several effects, the message reception probability in multiple sender scenarios is lower than in single sender-receiver scenarios. In case of an uncongested channel, the dominating phenomenon in multiple sender scenarios was found to be the hidden station effect [19].
This effect is caused by several sending nodes in the reach of a receiving node but with each sending node being out of the reach of the other sending nodes. In such constellations, the sending nodes are not able to coordinate the channel access and possibly send messages at the same time. This leads to colliding signals on the channel at the receiving node and hence decreases the probability for correct message reception.

The hidden station effect has multiple manifestations, but the dominant one comes into play after the hidden station range $d_{\mathrm{HS}}$ which is a section of the communication range $d_{\text {range }}^{\text {comm }}$. With a required signal-to-noise ratio threshold $\Gamma_{\text {SNR }}$ for successful decoding and a path loss coefficient $\mu$, we calculate $d_{\mathrm{HS}}$ as

$$
d_{\mathrm{HS}}=\frac{d_{\text {range }}^{\text {comm }}}{1+\left(\Gamma_{\mathrm{SNR}}\right)^{\frac{1}{\mu}}} .
$$

For constant $\mu, P_{\text {hidden }}^{\text {loss }}$ increases linearly with increasing distance [19]:

$$
P_{\text {hidden }}^{\text {loss }}(d, \rho) \approx 1.5 \cdot \rho \cdot \frac{T_{\text {air }}}{T_{\text {air }}+T_{\mathrm{CA}}} \cdot \frac{d-d_{\mathrm{HS}}}{d_{\mathrm{HS}}-1 \mathrm{~m}},
$$

where $T_{\text {air }}$ corresponds to the air time of a packet and $T_{\mathrm{CA}}$ corresponds to the channel access time.

\subsection{Combination of effects}

We combine $P_{\text {Nakagami }}^{\text {success }}$ and $P_{\text {hidden }}^{\text {loss }}$ as independent effects and calculate the message reception probability

$$
\begin{aligned}
& P_{\text {combined }}^{\text {success }}(d, \rho)= \\
& \left(1-P_{\text {hidden }}^{\text {loss }}(d, \rho)\right) \cdot P_{\text {Nakagami }}^{\text {success }}(d) .
\end{aligned}
$$

According to European standardization, congestion control will be implemented on multiple network layers, including the access layer [34]. Thus, the transmission of CAMs is governed by a congestion control mechanism whose objective is to keep the channel load low enough to guarantee efficient transmission. It takes effect at a relative channel load of $25 \%$ and above. Since our model does 
Table 2 Parameter configuration for the channel model

\begin{tabular}{lc}
\hline Parameter & Value \\
\hline$B_{\text {CAM }}$ & 200 byte \\
$C$ & $6 \frac{\text { Mbit }}{\mathrm{s}}$ \\
$\Gamma_{d_{\text {range }}^{\text {rct }}}$ & 10 \\
$T_{\text {air }}$ & $0.267 \mathrm{~ms}$ \\
$T_{\text {CA }}$ & $0.114 \mathrm{~ms}$ \\
$\mu$ & 2 \\
$h_{\text {snd }}, h_{\text {rCv }}$ & $1.5 \mathrm{~m}$ \\
$d_{\text {ramge }}^{\text {comm }}$ & $1,000 \mathrm{~m}$ \\
$\lambda$ & $0.0508 \mathrm{~m}$ \\
\hline
\end{tabular}

not respect congestion control effects, it is valid only up to a relative channel load of $25 \%$.

\subsection{Numerical results}

In our studies, we use the channel parameters provided in Table 2. For this set of parameters, Figure 8 shows the reception probability of a CAM depending on the distance $d$ between sender and receiver and on the relative channel load $\rho$. The reception probability decreases with the distance. It is almost independent of the studied channel load up to a distance of $250 \mathrm{~m}$. From then on, larger channel loads lead to lower CAM reception probabilities and the impact of the relative channel load on the reception probability is large, in particular in the range between 300 and $900 \mathrm{~m}$. Relative channel loads $\rho$ larger than $25 \%$ are not investigated because the applied channel model is not valid for that range. It would rather overestimate reception probabilities for such relative channel loads.

\section{Estimation of relative channel loads}

In the following, we first explain how the relative channel load is computed from logged CAM generation instants in the simulation. Then, we propose a simple formula that approximates the channel load depending on characteristic parameters. Finally, we discuss simulation results and compare them with analytical results. We show that the formula matches the simulated channel load quite well and use it to conduct a parameter study.

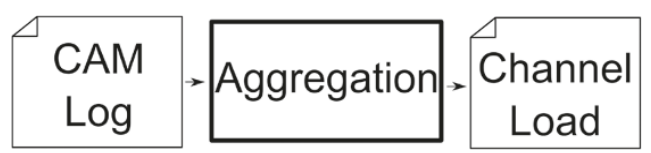

Figure 8 Illustration of the output of the channel model. The message reception probability depends on the distance between sender and receiver and the relative channel load. The model is only valid for relative channel loads $\rho \leq 25 \%$ as it ignores congestion control effects.

\subsection{Simulative approximation of relative channel loads} Figure 9 depicts our simulation tool chain for the determination of the relative channel load. The logged CAMs from Figure 2 are the input for an aggregation program which determines the local channel load for each receiving vehicle. We describe the performed steps in the following.

In Section 4, we used the output of the road traffic simulation to determine the instances at which vehicles would trigger CAMs. We now use these data in combination with the penetration rate of VANET technology to determine the rate $r_{\text {sent }}^{\mathrm{cr}}$ of sent CAMs in the vicinity of a receiver. The penetration rate $\varphi$ is the ratio of vehicles which are equipped with VANET technology.

We first determine which vehicles actually send CAMs in a specific time step. Then, we summarize the number of broadcasted CAMs within a range of $d_{\text {range }}^{\text {comm }}$ to determine the local rate of sent CAMs $r_{\text {sent }}^{\text {cr }}$ for each receiver and each simulation time step. Using $r_{\text {sent }}^{\mathrm{cr}}$ and Equation 3, we can calculate the perceived relative load $\rho$ for each receiver and each simulation time step. The mean relative channel load $\bar{\rho}$ is calculated as the average relative channel load over all simulated intervals and all receivers. We use it for validation and illustration purposes.

\subsection{Analytical approximation of relative channel loads}

To derive an analytical formula for the average relative channel load, we first calculate the average rate of messages triggered in the vicinity of each receiver by

$$
\bar{r}_{\mathrm{rcvd}}^{\mathrm{cr}}=2 \cdot n_{\text {lanes }} \cdot d_{\text {range }}^{\text {comm }} \cdot \sigma_{\text {vehicle }} \cdot \varphi \cdot \frac{1}{T_{\text {sent }}^{\mathrm{CAM}}} \text {. }
$$

This analytical approximation assumes straight roads, equidistant vehicles, and constant vehicle speeds leading to a constant time $T_{\text {sent }}^{\text {CAM }}$ between two consecutive CAMs from a single vehicle.

The average rate $\bar{r}_{\text {rcvd }}^{\text {cr }}$ of CAMs triggered in the vicinity of a receivers may be input to Equation 3 to calculate the average relative channel load.

\subsection{Numerical results}

Figure 10 depicts the average relative channel load from simulative and analytical approximation (circles and solid line, respectively) on the A5 and A6 scenarios. We observe, that the values depend about linearly on the penetration rate $\varphi$.

As mentioned before, our study is only valid for relative channel loads up to $\rho=25 \%$. That value is reached in the A5 scenario for a penetration rate of $\varphi=40 \%$ and in the A6 scenario for a penetration rate of $\varphi=80 \%$. For the A5 scenario, penetration rates $\varphi$ of $10 \%$ and $20 \%$ lead to relative channel loads of $6.25 \%$ and $12.5 \%$, respectively. The congestion control mechanism tries to mitigate channel congestion by adjusting several regulators like transmit power control, transmit rate control, transmit 


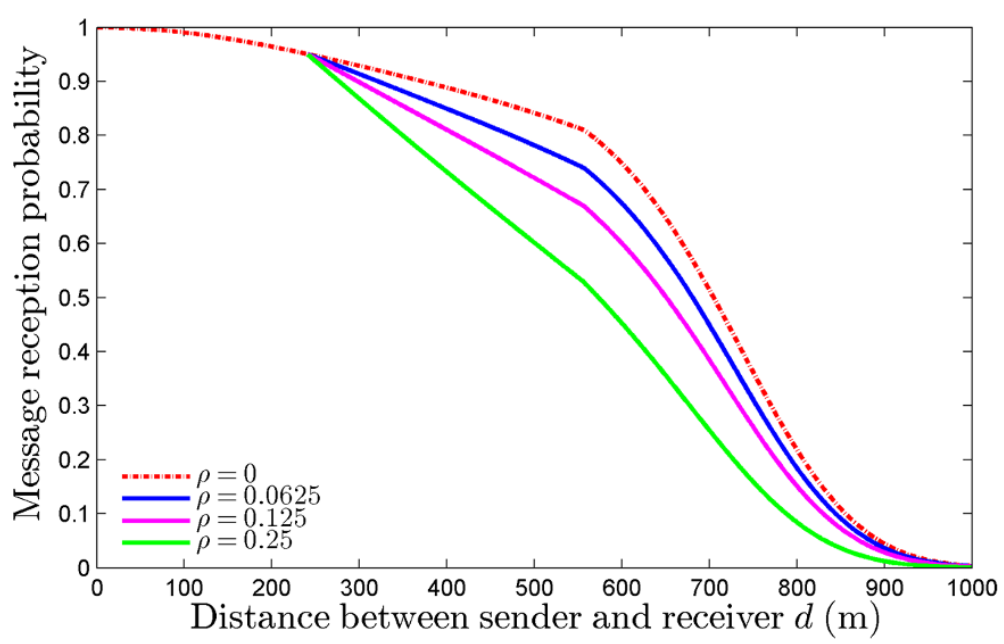

Figure 9 Tool chain to determine the channel load. The logged CAMs are aggregated over the receivers' vicinities to calculate their perceived relative channel load.

data rate control, and transmit access control. This effectively means that the transmission rate of a sender can be reduced which leads to message queuing, delay, and loss. Thus, CAM information may be outdated when it arrives at the receiver. To avoid such situations, the time between consecutive CAMs needs to be increased to generate them less frequently, which in turn decreases the surrounding awareness of the receiving vehicles. As we have not integrated congestion control in our simulative and analytical approximation, the relative channel loads larger than $25 \%$ are overestimated. From a relative load of $40 \%$ onwards, the wireless channel is even considered congested. We conclude that CAMs can fill the radio channel in the presence of moderate and high penetration rates in the future to such an extent that the available transmission capacity might not suffice to deliver all CAMs in time.

The solid line in Figure 10 shows the corresponding results approximated by Equations 9 and 3. We used $T_{\text {sent }}^{\text {CAM }}=0.2 \mathrm{~s}$ according to our results from Section 4.4 and $\sigma_{\text {vehicle }}=\frac{1}{35 \mathrm{~m}}$ for an average vehicle speed of $v=72 \frac{\mathrm{km}}{\mathrm{h}}$ according to the inter-vehicle distances described in Section 4.4.1. We observe that simulated

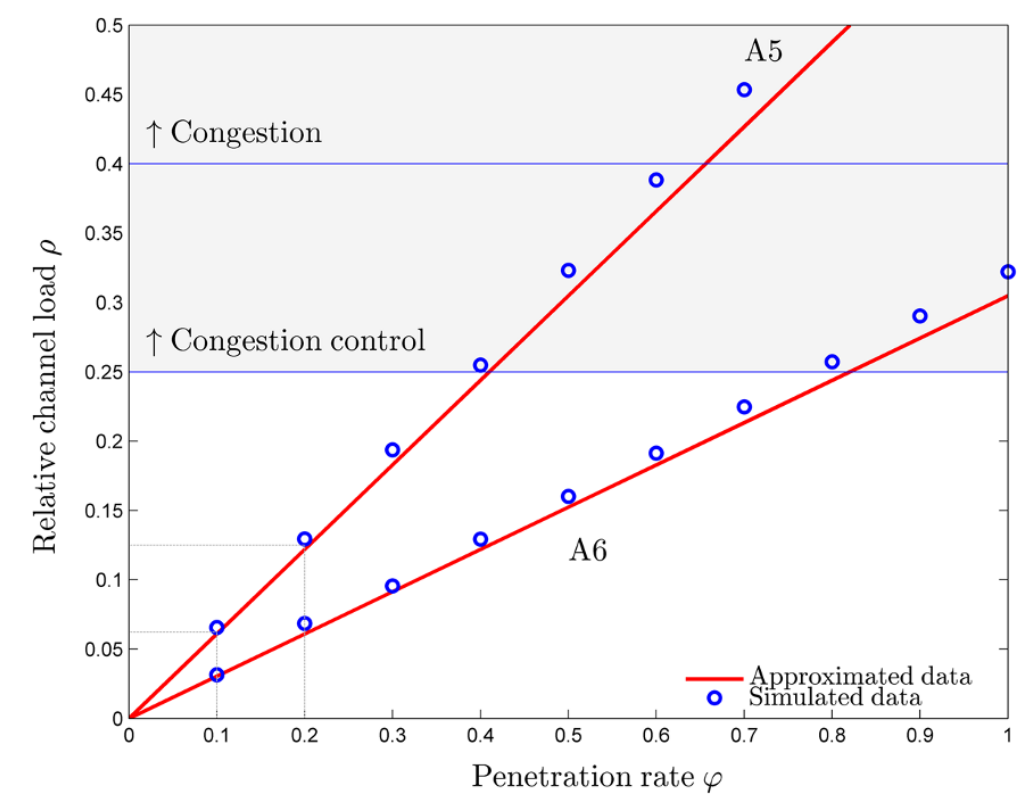

Figure $\mathbf{1 0}$ Relative channel loads in the $\mathbf{A} 5$ and $\mathbf{A} 6$ scenarios. Simulated and approximated relative channel loads for the A5 ( $\left.n_{\text {lanes }}=8\right)$ and the A6 $\left(n_{\text {lanes }}=4\right)$ scenarios. Relative channel loads above the congestion control threshold are overestimated. 


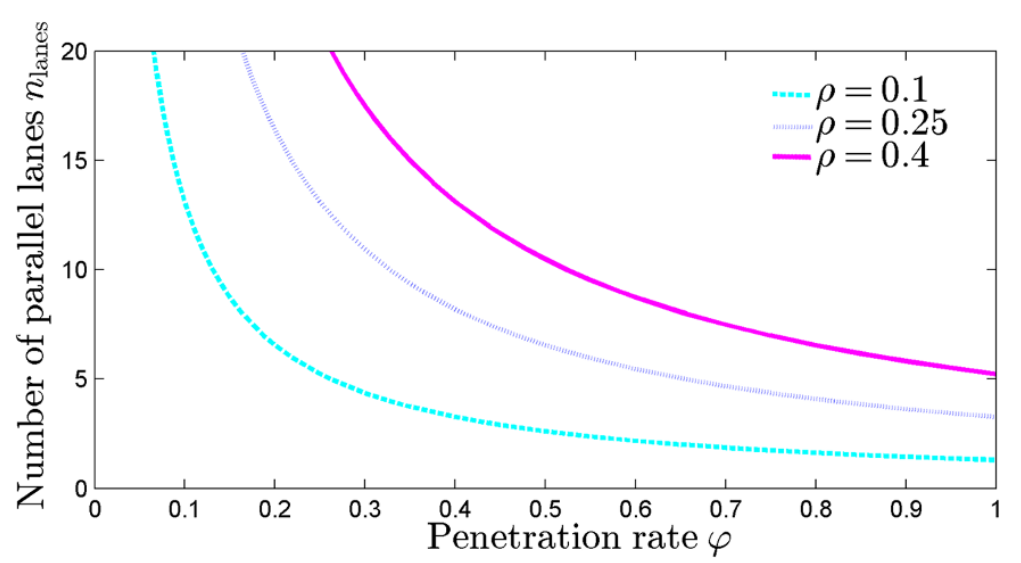

Figure 11 Conditions leading to a certain relative channel load. Penetration rate $\varphi$ and the number of parallel lanes $n_{\text {lanes }}$ determine the relative channel load $\rho$.

and approximated results are in good accordance. This validates the simulation results. This also recommends the analytical approximation formula for parameter studies.

We determine under which conditions a certain relative channel load can be achieved. Figure 11 shows the penetration rate $\varphi$ and the number of parallel lanes for which a relative channel load of $10 \%, 25 \%$, and $40 \%$ is achieved. We consider very large highways of up to 20 parallel lanes; such highways exist, e.g., Highway 401 in Toronto, Canada, which has 10 lanes in each direction. As our channel model is valid only up to a channel load of $\rho=25 \%$, the line for $\rho=40 \%$ is just a lower bound on the true values.

\section{Estimation of received CAM rates}

We use the presented channel model to estimate the received CAM rates for vehicles in various scenarios. Additionally, we estimate the number of CAMs a vehicle receives from other passing vehicles in the opposite direction. We use simulation and analytical approximation to obtain numerical results.

\subsection{Simulative approximation of received CAM rates}

Figure 12 depicts the used tool chain for the simulative approximation of received CAM rates. The triggered CAMs and the logged channel loads are input for a channel model function block which provides data for statistics.

The simulation in Section 4 determines all CAMs that are triggered and sent in a particular time step of $100 \mathrm{~ms}$. In Section 6, we derived the relative channel load at the position of each potential receiver. We compute the reception probability for each sender-receiver pair and for all logged CAMs. With this reception probability, we determine whether CAMs are successfully received by a vehicle. The corresponding CAM rate $r_{\text {rcvd }}^{\mathrm{CAM}}$ is computed by counting all received CAMs over 10 simulation steps which covers a duration of $1 \mathrm{~s}$, which yields the number of CAMs received by each vehicle in one second.

\subsection{Analytical approximation of received CAM rates}

We assume that all sent CAMs are equally distributed within the communication range of a receiver. The reception probability decreases with increasing distance to the sender. We further assume that the width of a lane is small compared to the communication range $d_{\text {range }}^{\text {comm }}$ and approximate the average reception probability for a CAM by

$$
\bar{P}_{\text {combined }}^{\text {success }}=\frac{\int_{0}^{d_{\text {range }}^{\text {comm }}} P_{\text {combined }}^{\text {success }} \mathrm{d} d}{d_{\text {range }}^{\text {comm }}} .
$$

Multiplication with the CAM rate in the communication range of a receiver yields

$$
r_{\text {rcvd }}^{\mathrm{CAM}}=\bar{r}_{\text {sent }}^{\mathrm{cr}} \cdot \bar{P}_{\text {combined }}^{\text {success }}
$$

as analytical approximation of the received CAM rate.

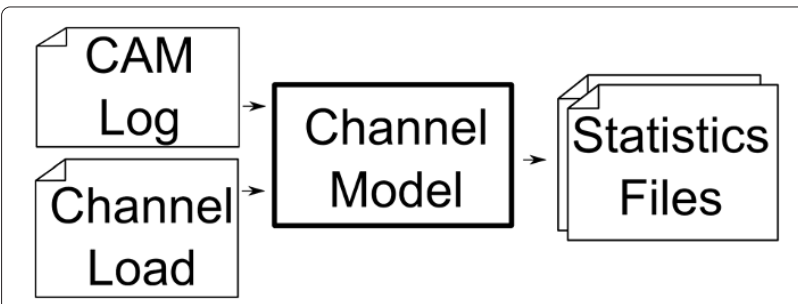

Figure 12 Tool chain to compute received CAM rates. The channel model computes a CAM reception probability with respect to the current channel load. It is used to simulate which of the generated CAMs are received by a vehicle. These data are gathered by statistics. 


\subsection{Numerical results}

We first analyze the received CAM rates in the A5 and A6 scenarios depending on the penetration rate. Then, we study the relative channel load and received CAM rates of vehicles in the A5 scenario for given vehicle speeds and penetration rates. Finally, we characterize under which conditions certain rates of received CAMs can be expected.

\subsubsection{Received CAM rates depending on penetration rates in the $A 5$ and $A 6$ scenarios}

Figure 13 illustrates the rates of CAMs received by vehicles on the A5 and A6 scenarios depending on the penetration rate $\varphi$. Analytically approximated data are given by solid lines and simulated mean values by circles with error bars showing the standard deviation. Maximum simulated values are given by small rectangles. Note that standard deviations and maximum values depend on the time frame over which we calculated the

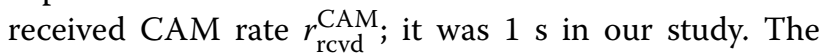
received CAM rates increase slightly less than linearly with the penetration rate for the same reason as discussed above. For CAM rates of up to $500 \frac{\mathrm{CAMs}}{\mathrm{s}}$, the channel model is sufficiently accurate due to the absence of congestion control. That CAM rate occurs in the A5 and the A6 scenarios for a penetration rate of $\varphi=$ $40 \%$ and $\varphi=80 \%$, respectively. Moreover, CAM rate peaks exceeding that boundary might appear even with congestion control being implemented because such a mechanism needs some time to take effect. We already observe high received CAM rates even for low penetration rates. In the A5 scenario, a penetration rate of $10 \%$ and $20 \%$ corresponds to relative channel loads of $6.25 \%$ and $12.5 \%$ which leads to up to 150 and $280 \frac{\mathrm{CAMs}}{\mathrm{s}}$, respectively.

\subsubsection{Received CAM rates depending on vehicle speeds in the A5 scenario}

We investigate the relative channel load and the received CAM rates for a penetration rate of $\varphi \in\{0.1,0.2,0.4,0.6\}$ and for vehicle speeds of $v=10 \frac{\mathrm{km}}{\mathrm{h}}$ and $v=72 \frac{\mathrm{km}}{\mathrm{h}}$ in the A5 scenario $\left(n_{\text {lanes }}=8\right)$. The $x$-axis in Figure 14 shows the obtained relative channel load, and the $y$-axis shows the received CAM rate for these data points. We first observe that the data points yield almost a line because the received CAM rate depends about linearly on the relative channel load. The red line indicates a straightly linear curve. However, the growth of the curve is less than linear for large relative channel loads $\rho$ because an increase of the relative channel load reduces the reception probability of CAMs. Due to the limitations of our channel model, only the data points with a relative channel load of at most $\rho=25 \%$ are valid. The lowest data point corresponds to $\varphi=0.1$ and the highest data point to $\varphi=0.6$ for each vehicle speed. We observe that slow traffic $\left(v=10 \frac{\mathrm{km}}{\mathrm{h}}\right)$ such as traffic jam leads to clearly lower channel load and received CAM rates than fast traffic $\left(v=72 \frac{\mathrm{km}}{\mathrm{h}}\right)$ in similar scenarios. This is due to the fact that the CAM rate density is largest for speeds around $v=72 \frac{\mathrm{km}}{\mathrm{h}}$ which has been illustrated in Figure 6. The A5 scenario comprises $n_{\text {lanes }}=8$ lanes, while the A6 scenario comprises only $n_{\text {lanes }}=4$ lanes. Therefore, the A6 scenario yields the

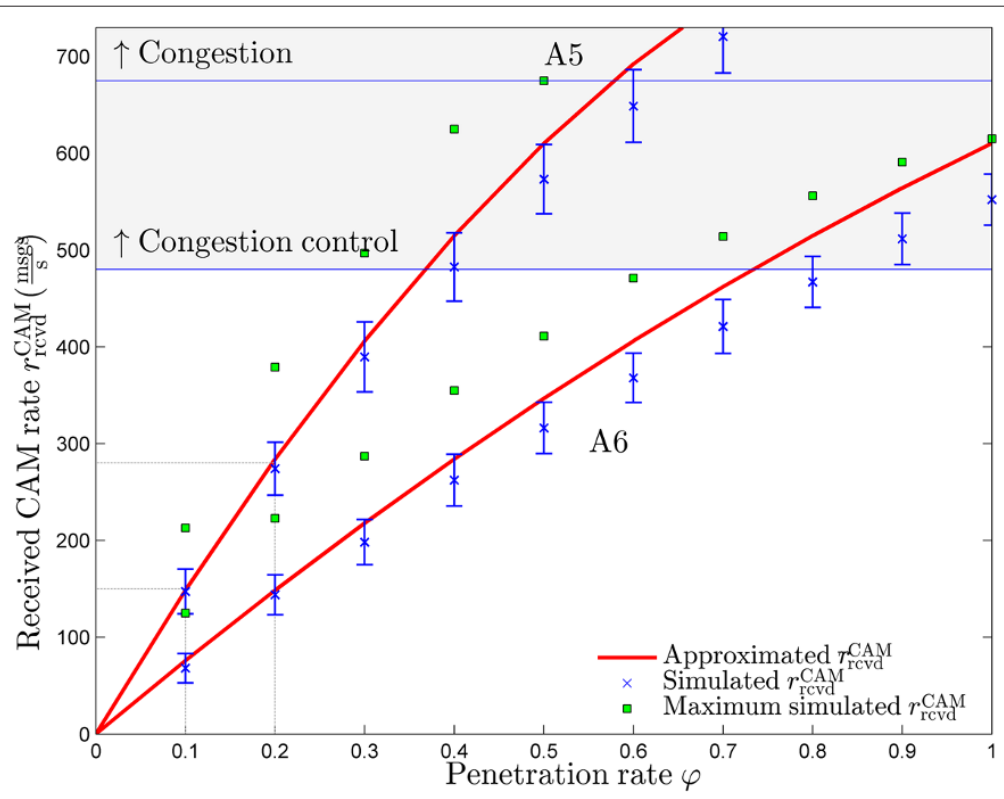

Figure 13 Rate of received CAMs in the $\mathbf{A} 5$ and $\mathbf{A} 6$ scenarios. Simulated and approximated received CAM rates for the A5 ( $\left.n_{\text {lanes }}=8\right)$ and the A6 $\left(n_{\text {lanes }}=4\right)$ scenarios. Received CAM rates above the congestion control threshold are overestimated. 


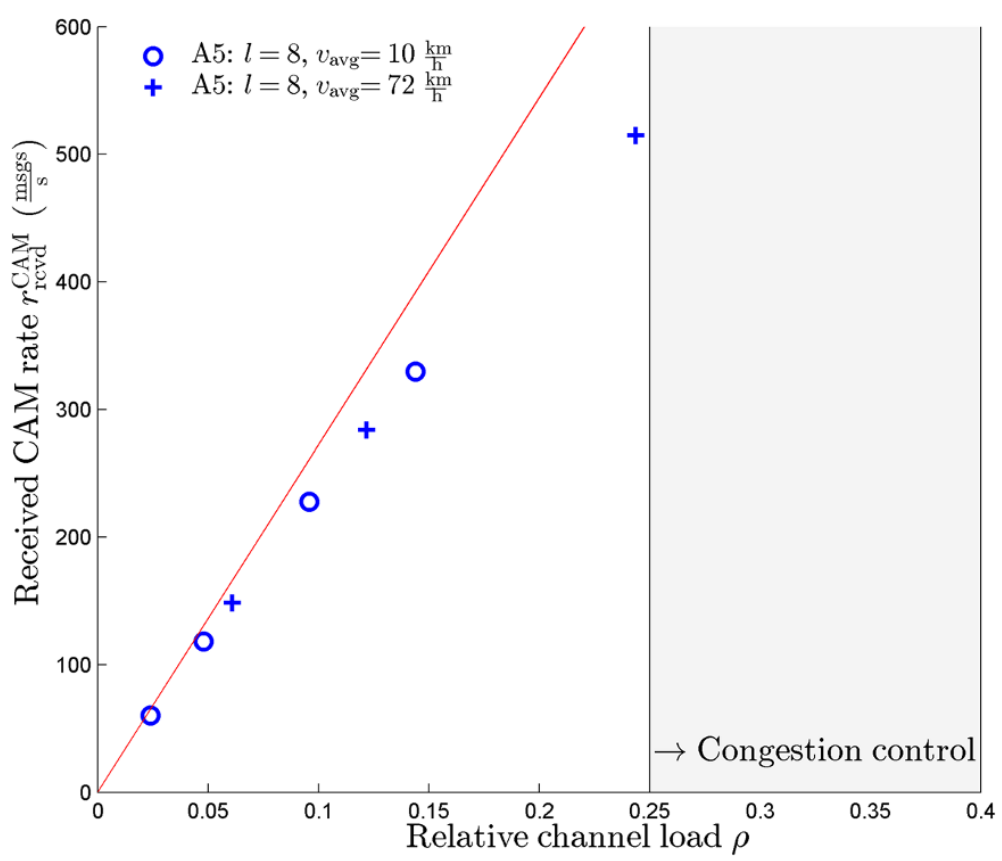

Figure 14 Relation between relative channel load and received CAM rate in the $\mathbf{A} 5$ scenario. Data points are provided for the A5 scenarios with $n_{\text {lanes }}=8$ lanes and penetration rates of $\varphi \in\{0.1,0.2,0.4,0.6\}$ and two different speeds. The lowest marker of each type corresponds to $\varphi=0.1$, the highest one to $\varphi=0.6$.

same data points for doubled values of the penetration rate $\varphi$. Therefore, we have omitted the results for the A6 scenario to avoid overlapping data points in the figure.

\subsubsection{General parameter study for received CAM rates}

We now evaluate under which conditions certain received CAM rates may be obtained. Figure 15 shows which penetration rates $\varphi$ and which number of lanes $n_{\text {lanes }}$ may lead to received CAM rates of $r_{\text {rcvd }}^{C A M} \in\{125,250,500\}$ $\frac{\mathrm{CAMs}}{\mathrm{s}}$. On roads with only two lanes, 125 and $250 \frac{\mathrm{CAMs}}{\mathrm{s}}$ are obtained only for a penetration rate of $33 \%$ and $70 \%$, respectively, which is rather high. However, on highways with eight lanes, 125,250 , and $500 \frac{\mathrm{CAMs}}{\mathrm{s}}$ are already reached for penetration rates of $8 \%, 18 \%$, and $40 \%$, a situation that may be faced in the next years.

We conclude that even for low penetration rates, series vehicles equipped with VANET technology according to current standardization have to handle several hundreds of messages per second under challenging conditions. Considering the usual restrictions for automotive hardware and software regarding robustness, size, and cost,

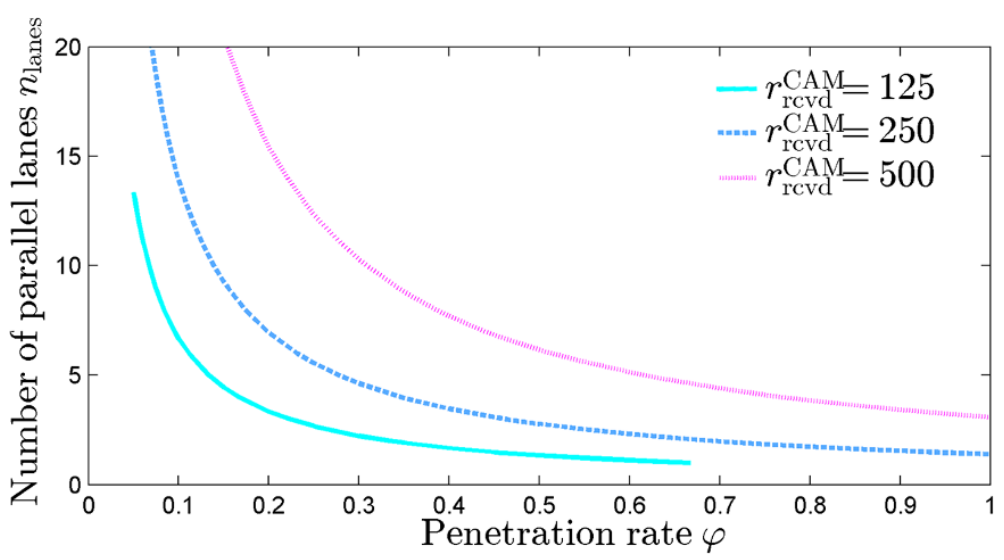

Figure 15 Conditions leading to a certain received CAM rate. Penetration rate $\varphi$ and number of parallel lanes $n_{\text {lanes }}$ determine the received CAM rate $r_{\text {rCVd }}^{\text {CAM }}$. 
there are two ways to deal with this challenge, with the option to combine both of them. First, the hardware capabilities are dimensioned sufficiently high which in turn leads to higher costs and potentially larger size. Second, the CAM processing chain is designed sufficiently smart. For instance, the CAM processing engine may serve most important CAMs preferably so that only least important CAMs are discarded. We proposed such an algorithm in [35].

\subsection{Number of CAMs received from passing vehicles}

The number of received CAMs received from specific vehicles depends on the sender's and receiver's trajectories. If both vehicles drive on the same lane in the same direction at the same speed, the number of CAMs transferred between them depends on how long they drive past each other. In contrast, if both vehicles pass each other in opposite direction, there is only a short time window within which they can exchange CAMs. In the following, we determine the number $n_{\text {rcvd }}^{\text {passing }}$ of CAMs received by a vehicle from another vehicle passing in opposite direction depending on the speed of both vehicles. To reduce the parameter range, we assume constant and equal speed for both vehicles.

The time $t_{\text {pass }}$ during which a sender stays in the receiver's communication range $d_{\text {range }}^{\text {comm }}$ while passing by at an average vehicle speed $v$ can be calculated by

$$
t_{\text {pass }}=\frac{d_{\text {range }}^{\text {comm }}}{2 \cdot v} \text {. }
$$

The number of received CAMs from a passing vehicle $n_{\text {rcvd }}^{\text {passing }}$ can be approximated by

$$
n_{\mathrm{rcvd}}^{\text {passing }}=\frac{t_{\text {pass }}}{T_{\mathrm{CAM}}^{\text {inter }}} \cdot \bar{P}_{\text {combined }}^{\text {success }} .
$$

Figure 16 illustrates the number of CAMs $n_{\mathrm{rcvd}}^{\text {passing }}$ received from a single vehicle that is passing by in opposite direction. We assumed a four-lane scenario like in our example of the A6, and we considered average vehicle speeds $v$ between 0 and $150 \frac{\mathrm{km}}{\mathrm{h}}$.

For speeds lower than $10 \frac{\mathrm{km}}{\mathrm{h}}$, very high numbers of CAMs are received from each passing vehicle. This results from a long passing time $t_{\text {pass }}$ in slow traffic with the maximum CAM inter-generation time of $1 \mathrm{~s}$ at low speed. For typical highway speeds, a vehicle exchanges between 40 and 80 CAMs while passing by. This range is relatively stable as the higher CAM generation rates compensate the lower communication window $t_{\text {pass }}$ while passing.

Not all CAMs from passing vehicles are of equal utility. It is obvious that for most applications, only the CAMs originating from positions ahead of the receiver contain useful information. As a consequence, only half of the received CAMs is of particular interest. During high-load conditions where not all CAMs can be processed, the CAMs originating from positions ahead of the receiver should be preferably processed.

\section{Origin of received CAMs}

From VANET applications point of view, the relevance of CAMs depends on distance and direction of their senders with regard to the receiver, and these relevances depend on the specific application. While crash avoidance applications need information about the near vicinity of the receiving car, traffic jam detection applications analyze an area a few hundred meters or kilometers ahead of the receiving car. For the design of these applications, the distribution of the origin of received CAMs relative to the receiver is useful input.

In this section, we characterize the distance of senders from which vehicles receive CAMs. We explain our simulation approach and derive analytical expressions for those data. Then, we discuss the results for a sample parameter set.

\subsection{Simulative approximation of origin of received CAMs}

We simulate the reception of CAMs and collect statistics about the distance of their sender and receiver. Therefore, we define distance intervals $\left[d_{1}, d_{2}\right]$ of 100 -m lengths which are the base for a histogram that provides the fraction of CAMs that originate from those distance intervals. For our evaluation, we rather use the cumulative histogram that accumulates the relative frequencies in the normal histogram up to a certain distance interval.

\subsection{Analytical approximation of origin of received CAMs}

The success probability $P_{\text {combined }}^{\text {success }}(d, \rho)$ governs whether a vehicle receives a CAM that is generated by another vehicle which is $d$ away while the vehicle experiences a relative channel load $\rho$. Thus, the distribution function of the distances of vehicles from which CAMs are received can be computed by

$$
P(D \leq d, \rho)=\frac{\int_{0}^{d} P_{\text {combined }}^{\text {success }}(d, \rho) \mathrm{d} d}{\int_{0}^{d_{\text {range }}^{\text {connge }}} P_{\text {combined }}^{\text {success }}(d, \rho) \mathrm{d} d} .
$$

\subsection{Numerical results}

We study the distribution function of the origin of CAMs in the A5 and A6 scenarios. We collect data about CAMs received within the boxed areas in both scenarios that are depicted in Figures 3 and 4. Vehicles in this area receive CAMs only from vehicles ahead or behind them, but not from vehicles driving on other roads.

Figure 17 shows the analytical and empirical cumulative distribution of the origin of CAMs relative to the receiver. The curves are gained from simulation and analysis of the A5 and A6 scenarios assuming a penetration rate 


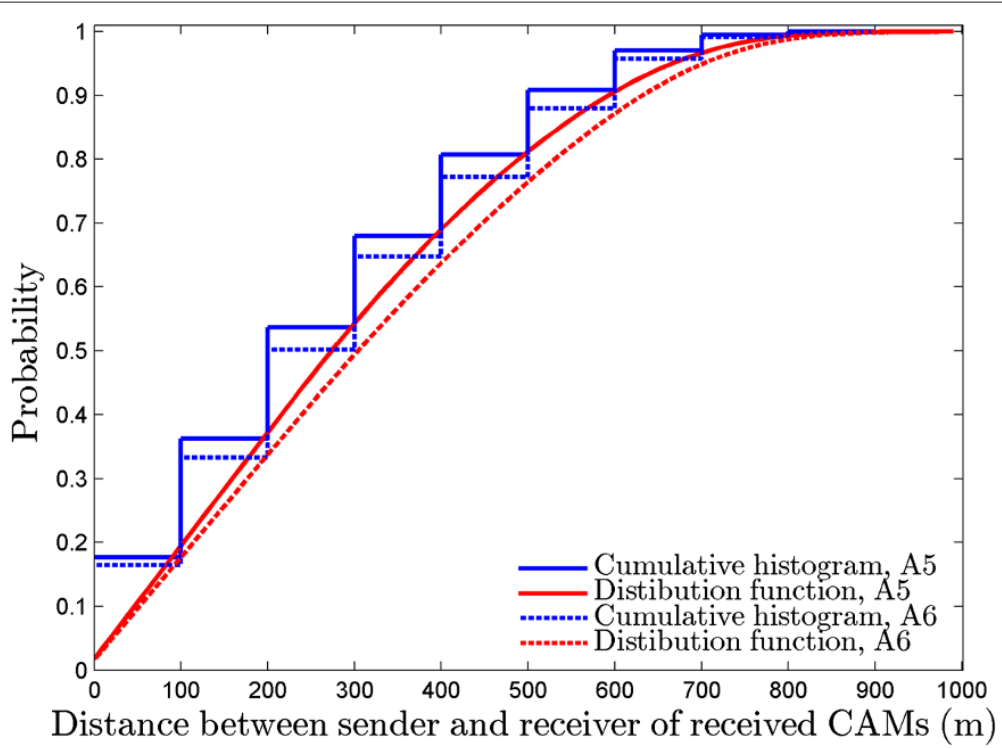

Figure 16 Number of received CAMs per passing vehicle $\boldsymbol{n}_{\text {rcvd }}^{\text {passing }}$. The number $n_{\text {rcvd }}^{\text {pasing }}$ of CAMs received from a single vehicle passing by in opposite direction depends on the average vehicle speed $v$. A highway with two lanes per direction was assumed for the analysis.

of $\varphi=40 \%$. The analytical curves match the simulated curves quite well so that the analytical approach may be used for estimation of the percentage of received CAMs originating from within a specific distance.

We observe that the distribution functions for the A5 scenario approach large probabilities faster than those for the A6 scenario. This means that in the A5 scenario, a larger fraction of CAMs is received from the near vicinity than in the A6 scenario. While $90 \%$ of the CAMs are received from other vehicles within a distance of up to $600 \mathrm{~m}$ in the $\mathrm{A} 5$ scenario, this $90 \%$ quantile is about $650 \mathrm{~m}$ in the A6 scenario. The reason for the difference is the fact that the eight lanes in the A5 scenarios cause a larger channel load than the four lanes in the A6 scenario. As a result, CAMs from faraway senders in the A5 scenario have a clearly lower reception probability than in the A6 scenario.

The information that only a minority of CAMs comes from faraway vehicles is important for the design of VANET applications that use the local dynamic map (LDM). The LDM can only be precise for the area from which the most messages are received.

If the CAM processing unit is not able to process all received CAMs, some CAMs need to be discarded. Depending on the application, CAMs from nearby vehicles may be more important than CAMs from faraway vehicles or vice versa. If CAMs received from faraway vehicles are especially useful, most of them may be delivered to applications if CAMs from nearby vehicles are preferably dropped. If CAMs received from nearby

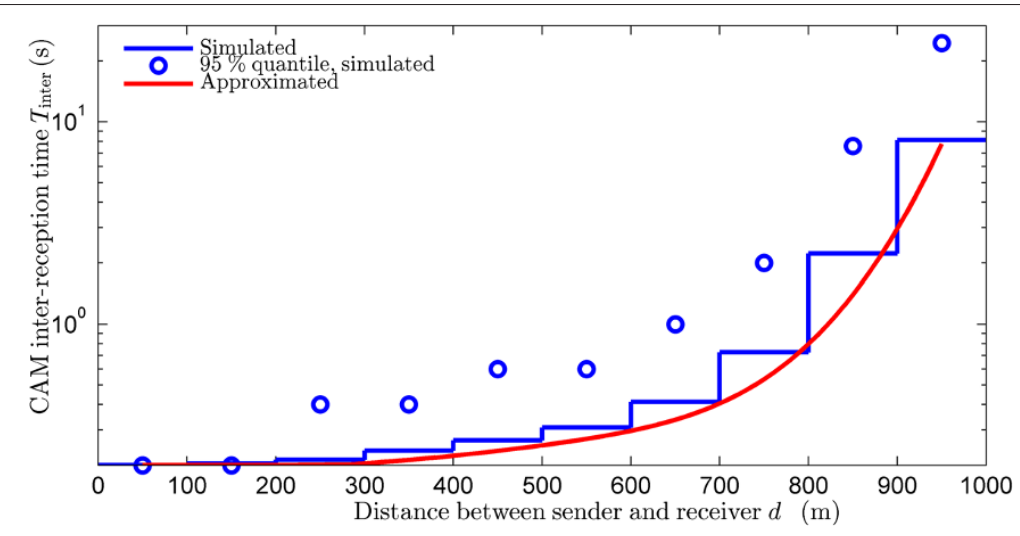

Figure 17 Origin of received CAMs. Comparison of the simulated cumulative histogram and the analytically computed distribution function; A5 scenario with $n_{\text {lanes }}=8$ lanes, $\varphi=40 \%$; A6 scenario with $n_{\text {lanes }}=4$ lanes, $\varphi=40 \%$. 
vehicles are especially useful, dropping CAMs from faraway vehicles reduces the rate of received CAMs only slightly so that CAMs from nearby vehicles also need to be dropped.

\section{Estimation of CAM inter-reception times}

Information carried by CAMs will be merged with information from in-car sensors to enable (semi-) autonomous vehicular functions. In contrast to in-car sensors like radar and image recognition, VANETs cannot guarantee strictly regular CAM updates due to the unreliability of the wireless channel and its inherent message transmission failures. Information about average and bad-case CAM inter-reception times from specific vehicles is a crucial input for the design of data fusion algorithms which combine VANET data and in-car sensor information.

In this section, we first describe a simulative and an approximative analytical approach to derive CAM inter-reception times depending on the distance between sender and receiver. Then, we discuss numerical results which show among others that CAMs from faraway vehicles are received very rarely. This is important input for filter algorithms that select most important CAMs if the CAM processing unit is overloaded.

\subsection{Simulative approximation of CAM inter-reception times}

We simulate CAM inter-reception times $T_{\left[d_{1}, d_{2}\right]}^{\mathrm{inter}}$ depending on the distance between sender and receiver. To that end, we $\log$ for each receiver the last received CAM from any sender and the distance $d$ to that sender so that inter-reception times can be computed at the reception of new CAMs. We collect statistics for inter-reception times depending on distance between sender and receiver. Again, we use distance intervals $\left[d_{1}, d_{2}\right]$ of $100-\mathrm{m}$ lengths for that purpose.
We collect inter-reception times only if the distance between sender and receiver falls in the same distance interval for the currently and the previously received CAM.

\subsection{Analytical approximation of CAM inter-reception times}

We now determine average inter-reception times $E\left[T_{d}^{\text {inter }}\right]$ using our statistical channel model for a given senderreceiver distance $d$.

The time between consecutive CAMs received from the same sender is a random variable that can be modeled by a geometric distribution with a shift of $1(\operatorname{GEOM}(1))$ :

$$
\begin{aligned}
P\left(T_{d}^{\text {inter }}\right. & \left.=i \cdot T_{\text {sent }}^{\text {CAM }}\right)= \\
& \left(1-P_{\text {combined }}^{\text {success }}(d, \rho)\right)^{(i-1)} \cdot P_{\text {combined }}^{\text {success }}(d, \rho),
\end{aligned}
$$

where $T_{\text {sent }}^{\mathrm{CAM}}$ is the average time between two CAMs generated by the sender. The mean of the random variable $T_{d}^{\text {inter }}$ is given by

$$
E\left[T_{d}^{\mathrm{inter}}\right]=\sum_{i=1}^{\infty i \cdot T_{\mathrm{sent}}^{\mathrm{CAM}} \cdot P\left(T_{d}^{\mathrm{inter}}=i \cdot T_{\mathrm{sent}}^{\mathrm{CAM}}\right)}
$$

In contrast to the simulation, the analysis does not consider vehicle movements.

\subsection{Numerical results}

We investigate CAM inter-reception times for the A5 scenario and a penetration rate of $\varphi=20 \%$. Figure 18 illustrates analytical values according to Equation 16 depending on the distance between sender and receiver. It also shows simulated mean values of inter-reception times for each section together with $95 \%$ quantiles. We observe that mean inter-reception times stay near the minimum of one CAM inter-generation time $T_{\text {sent }}^{\mathrm{CAM}}$ up to a distance

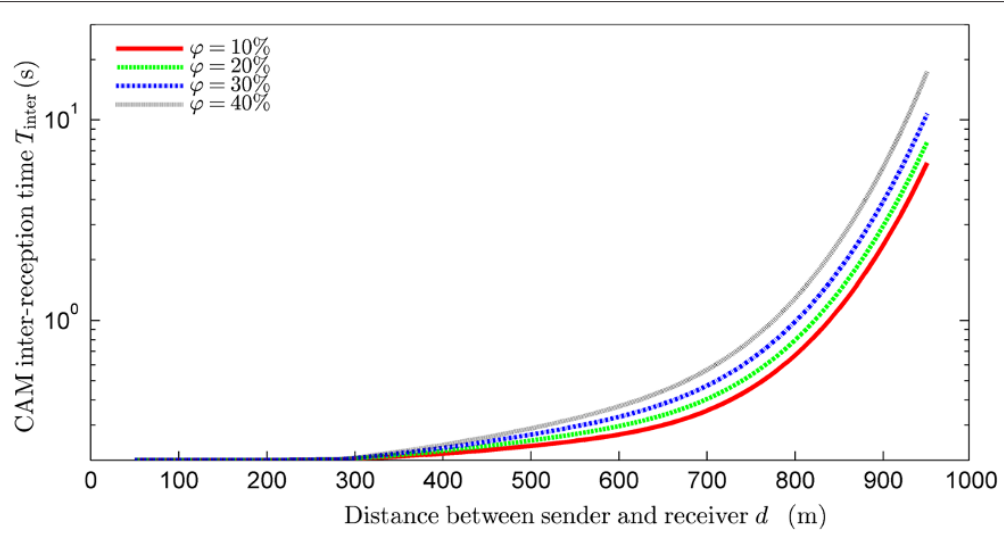

Figure 18 Inter-reception times of CAMs. Inter-reception times of CAMs by distance between sender and receiver. Comparison of simulation (steps $=$ mean values; circles $=$ maximum values) and analytical results (A5 scenario, $\varphi=20 \%$ ). 


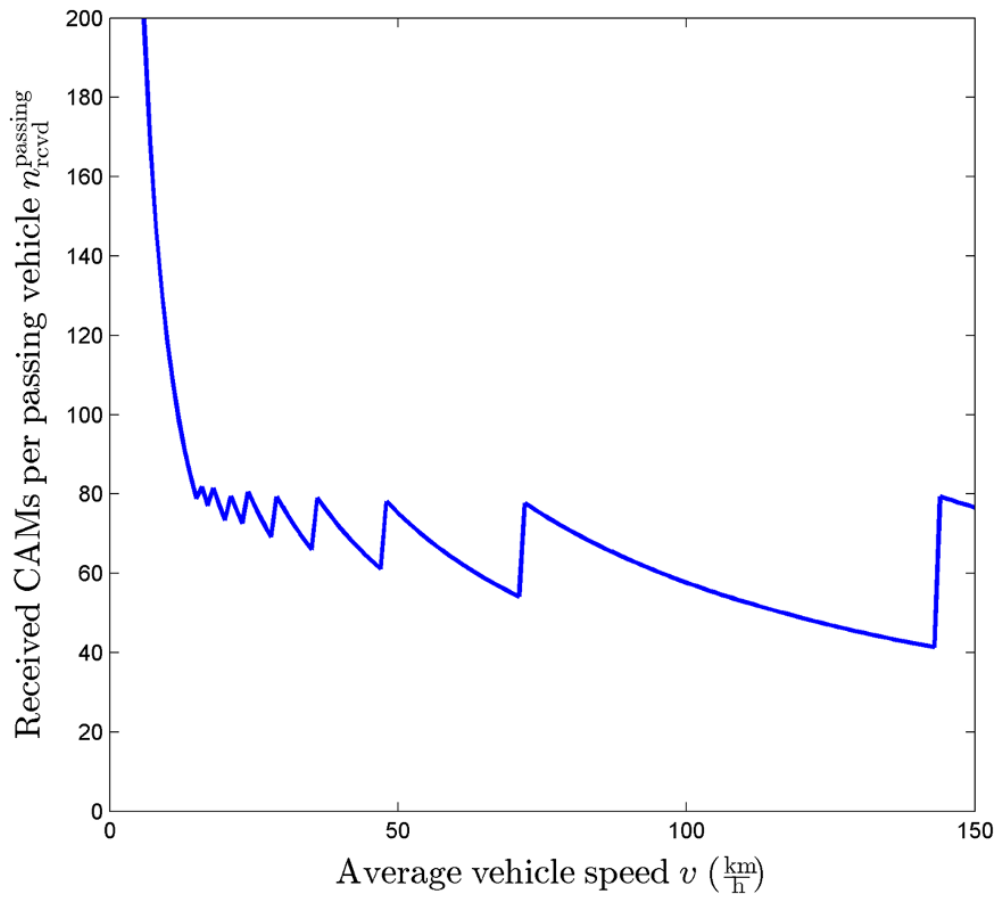

Figure 19 Inter-reception times of CAMs. Inter-reception times of CAMs by distance between sender and receiver. Comparison of analytical results for different penetration rates in the $\mathrm{A} 5$ scenario.

of $500 \mathrm{~m}$ between sender and receiver. That means, only a few messages are lost on the channel. For $500 \mathrm{~m}$ and above, the average inter-reception time increases clearly with distance and is about $8 \mathrm{~s}$ for senders which are 900 to $1,000 \mathrm{~m}$ away from the receiver. The analytical calculation fits the simulative results, which validates the simulation. The $95 \%$ quantiles of inter-reception times are close to the mean values for distances up to $200 \mathrm{~m}$; beyond that distance, the $95 \%$ quantile of the inter-reception time is significantly higher than its mean value.

The results indicate that applications with interest in CAMs from nearby vehicles, e.g., collision mitigation applications, can expect to frequently receive CAMs from vehicles of interest. In contrast, applications with interest in CAMs from faraway vehicles must respect that they rarely receive CAMs from vehicles of interest. This may be taken into account for relevance estimation of CAMs in smart CAM processing engines: a CAM from a faraway vehicle may be considered more valuable than a CAM from a nearby vehicle.

Our analysis is based on continual traffic flows leading to almost constant CAM inter-generation times $T_{\text {sent }}^{\mathrm{CAM}}$. If the traffic flow is less continual, other CAM intergeneration times may lead to other CAM inter-reception times. In our simulation, the traffic flow and channel model were chosen to produce high channels loads and received CAM rates. These parameters lead to rather low inter-reception times; inter-reception times are higher for pessimistic channel conditions and/or a curvy road networks with obstacles. If VANET applications require estimates of CAM inter-reception times, more results are needed for realistic or pessimistic conditions that may lead to larger values.

Finally, we study how inter-reception times depend on the penetration rate. Figure 19 shows the inter-reception times for different sender-receiver distances and penetration rates. For small distances, there is hardly any difference in inter-reception time. However, for large distances, the inter-reception time increases with the penetration rate. Thus, applications leveraging information from faraway vehicles may work better in the beginning of VANET deployment than in stages with high penetration rate.

\section{Conclusions}

In this paper, we studied the transmission of Cooperative Awareness Messages (CAM) in future VANETs. We used a newly developed simulation tool chain with a simplified channel model that is valid as long as congestion control in the access layer does not take effect.

We simulated two realistic road traffic scenarios to analyze rates of sent CAMs, relative channel loads, received CAM rates, the number of CAMs from a vehicle passing in opposite direction, the origin of received CAMs, and the frequency of CAMs from vehicles in a certain distance. We validated all simulation results with analytical approximation formulae that may also be used for 
parameter studies. In addition, we performed parameter studies based on the approximation formulae to understand under which conditions certain channel loads or CAM rates may appear.

The evaluation shows that the relative channel load can be large enough to cause congestion on the radio channel so that congestion mechanisms for CAM generation are required. This holds even for moderate penetration rates of VANET technology. The rate of received CAMs can easily reach $500 \frac{\text { CAMs }}{\mathrm{s}}$ which future series vehicles need to handle. This requires either costly hardware with sufficient processing power and expensive high-bandwidth, low-latency in-vehicle networks or a smart CAM processing engine that preferably treats most important CAMs and discards least important CAMs if processing capacity does not suffice in the presence of high received CAM rates. A vehicle receives about 40 to 100 CAMs from vehicles that pass by in opposite direction, but only half of them carry information of interest due to their location. Most received CAMs originate from senders within $600 \mathrm{~m}$ from the receiver. Therefore, the information density is high for low-distance regions around a receiver. As a consequence, the time between CAMs from a single sender may be very large for faraway senders.

\section{Competing interests}

The authors declare that they have no competing interests.

\section{Acknowledgements}

We thank Christian Weiss and Wilfried Enkelmann for fruitful discussions and their support. We acknowledge support by the Deutsche

Forschungsgemeinschaft and the Open Access Publishing Fund of Tuebingen University.

\section{Disclosure}

This journal version is an extension of our prior work 'Analysis of Cooperative Awareness Message Rates in VANETs' published in the Proceedings of the 13th International Conference on Intelligent Transportation Systems

Telecommunications 2013.

Received: 1 February 2014 Accepted: 6 June 2014

Published: 17 June 2014

\section{References}

1. M Sichitiu, M Kihl, Inter-vehicle communication systems: a survey. IEEE Communications Surveys \& Tutorials. 10(2), 88-105 (2008)

2. CWeiß, V2X Communication in Europe - from research projects towards standardization and field testing of vehicle communication technology. Computer Networks. 55(14), 3103-3119 (2011)

3. European Telecommunications Standards Institute (ETSI), Intelligent Transport Systems (ITS); Vehicular Communications; Basic Set of Applications; Part 3: Specifications of Decentralized Environmental Notification Basic Service, Technical Specification (102 637-3) (ETSI, Nice, 2010)

4. European Telecommunications Standards Institute (ETSI), Intelligent Transport Systems (ITS); Vehicular Communications; Basic Set of Applications; Part 2: Specification of Cooperative Awareness Basic Service, Technical Specification (302 637-2) (ETSI, Nice, 2011)

5. CAR 2 CAR Communication Consortium, Memorandum of understanding for OEMs within the CAR 2 CAR Communication Consortium on Deployment Strategy for Cooperative ITS in Europe (2011). http://www. car-to-car.org/index.php?id=11. Accessed 11 June 2014
6. $\mathrm{ABI}$ Research, V2V Penetration in New Vehicles to Reach $62 \%$ by 2027 (2013). http://www.abiresearch.com/press/v2v-penetration-in-newvehicles-to-reach-62-by-202. Accessed 11 June 2014

7. Kraftfahrt-Bundesamt, Fahrzeugzulassungen (FZ); Bestand an Kraftfahrzeugen und Kraftfahrzeuganhängern nach Fahrzeugalter; 1. Januar 2012 (2012)

8. H Stubing, M Bechler, D Heussner, T May, I Radusch, H Rechner, P Vogel, simTD: a car-to- $X$ system architecture for field operational tests. IEEE Communications Magazine. 48(5), 148-154 (2010)

9. J-H Wilbrod, G Segarra, Système Coopératif Routier Expérimental France (2012)

10. A Festag, L Le, M Goleva, Field operational tests for cooperative systems: a tussle between research, standardization and deployment. Paper presented at the 8th ACM international workshop on vehicular inter-networking, Las Vegas, 19-23 Sept 2011 pp. 73-78

11. C Sommer, R German, F Dressler, Bidirectionally coupled network and road traffic simulation for improved IVC analysis. IEEE Trans. Mobile Comput. 10(1), 3-15 (2011)

12. B Schünemann, V2X simulation runtime infrastructure VSimRTI: an assessment tool to design smart traffic management systems. Computer Networks: The International Journal of Computer and Telecommunications Networking. 55(14), 3189-3198 (2011)

13. SUMO Simulation of Urban MObility (2013). http://sumo.sourceforge.net/. Accessed 11 June 2014

14. V Shivaldova, Mecklenbräuker CF, Real-world measurements-based evaluation of IEEE $802.11 \mathrm{p}$ system performance. Paper presented at the 5th international symposium on wireless vehicular communications. Dresden, 2-3 June 2013

15. A Taimoor, B Laura, A Thiel, CF Mecklenbräuker, F Tufvesson, Measurements based channel characterization for vehicle-to-vehicle communications at merging lanes on highway. Paper presented at the 5th international symposium on wireless vehicular communications. Dresden, 2-3 June 2013

16. M Killat, $\mathrm{H}$ Hartenstein, An empirical model for probability of packet reception in vehicular ad hoc networks. EURASIP Journal on Wireless Communications and Networking. 2009, 4-1412 (2009)

17. M Killat, F Schmidt-Eisenlohr, H Hartenstein, C Rössel, P Vortisch, S Assenmacher, F Busch, Enabling efficient and accurate large-scale simulations of VANETs for vehicular traffic management. Paper presented at the 4th ACM international workshop on vehicular ad hoc networks. Montreal, 10 Sept 2007

18. V Taliwal, D Jiang, H Mangold, C Chi, R Sengupta, Empirical determination of channel characteristics for DSRC vehicle-to-vehicle communication. Paper presented at the 1st ACM international workshop on vehicular ad hoc networks. Philadelphia 1 Oct 2004

19. A Brakemeier, White paper on network design limits and VANET performance. (Car 2 Car Communication Consortium, Braunschweig, 2009)

20. M Torrent-Moreno, D Jiang, $\mathrm{H}$ Hartenstein, Broadcast reception rates and effects of priority access in 802.11-based Vehicular Ad-hoc Networks. Paper presented at the 1st ACM international workshop on vehicular ad hoc networks, Philadelphia. 1 Oct 2004

21. R Reinders, M van Eenennaam, G Karagiannis, G Heijenk, Contention window analysis for beaconing in VANETs. Paper presented at the 7th IEEE international wireless communications and mobile computing conference, Istanbul. 5-8 July 2011

22. K Lam, Design of a medium access control protocol that exploits the structures of vehicular networks, Dissertation, Urbana-Champaign (2011)

23. A Bin Tariq, vulnerable vehicles problem. Paper presented at the 11 th conference of open innovations Association-Finnish-Russian University Cooperation in Telecommunications, St. Petersburg. 23-27 April 2012

24. European Telecommunications Standards Institute (ETSI), Intelligent Transport Systems (ITS); Harmonized Channel Specifications for Intelligent Transport Systems Operating in the $5 \mathrm{GHz}$ Frequency Band, Technical Specification (102 724). (ETSI, Nice, 2012)

25. Car 2 Car Communication Consortium, The Handbook for Vehicle-to-X Cooperative Systems Simulation. (Car 2 Car Communication Consortium, Braunschweig, 2011)

26. 'TAPAS Cologne' scenario (2011). http://sumo.sourceforge.net/doc/ current/docs/userdoc/Data/Scenarios/TAPASCologne.html. Accessed 11 June 2014 
27. A Wegener, M Piórkowski, M Raya, H Hellbrück, S Fischer, J-P Hubaux, TraCl: An interface for coupling road traffic and network simulators. Paper presented at the 11 th communications and networking simulation symposium. Ottawa, 14-17 April 2008

28. SUMO traffic modeler (2013). http://sourceforge.net/projects/ trafficmodeler/. Accessed 11 June 2014

29. B Filzek, Abstandsverhalten Auf Autobahnen. (Dissertation, TU Darmstadt, 2007)

30. European Telecommunications Standards Institute (ETSI), Intelligent Transport Systems (ITS); European Profile Standard for the Physical and Medium Access Control Layer of Intelligent Transport Systems Operating in the $5 \mathrm{GHz}$ Frequency Band, Standard (202 663). (ETSI, Nice, 2010)

31. N Nakagami, The m-distribution, a general formula for intensity distribution of rapid fading, in Statistical Methods in Radio Wave Propagation (Pergamon Press Oxford, p. 1960

32. C Sommer, F Dressler, Using the right two-ray model? A measurement based evaluation of PHY models in VANETs. Paper presented at the 17th ACM international conference on mobile computing and networking, Las Vegas. 19-23 Sept 2011

33. TS Rappaport, Wireless Communications: Principles and Practice (2nd Edition). Prentice Hall, Austin, 2001)

34. European Telecommunications Standards Institute (ETSI), Intelligent Transport Systems (ITS); Decentralized Congestion Control Mechanisms for Intelligent Transport Systems Operating in the $5 \mathrm{GHz}$ Range; Access Layer Part, Technical Specification (102 687). (ETSI, Nice, 2011)

35. J Breu, M Menth, Relevance estimation of Cooperative Awareness Messages in VANETs. Paper presented at the 5 th international symposium on wireless vehicular communications. Dresden, 2-3 June 2013

doi:10.1186/1687-1499-2014-98

Cite this article as: Breu et al:: A quantitative study of Cooperative Awareness Messages in production VANETs. EURASIP Journal on Wireless Communications and Networking 2014 2014:98.

\section{Submit your manuscript to a SpringerOpen ${ }^{\mathcal{O}}$ journal and benefit from:}

- Convenient online submission

- Rigorous peer review

- Immediate publication on acceptance

- Open access: articles freely available online

- High visibility within the field

- Retaining the copyright to your article

Submit your next manuscript at $\boldsymbol{\wedge}$ springeropen.com 